\section{NHS Health Checks in a primary care dental setting - an opportunity for the profession to maximise uptake for public health partners?}

\author{
M. G. McGrady, ${ }^{1 *}$ J. Pickford, ${ }^{2}$ E. Hawthorn, ${ }^{3}$ J. Waterall ${ }^{4}$ and C. Bridgman ${ }^{5}$
}

IN BRIEF

- Discusses the need for dental teams to embrace and engage with healthcare or public health partnerships.

- Presents innovative approaches, involving multi-agencies, to improve the uptake of NHS Health Checks.

- Shows how skill-mix and working practices of dentists could potentially reach target populations and maximise efficiency and uptake of NHS Health Checks.

This opinion piece considers an opportunity for primary dental care practitioners to work in partnership with public health teams to maximise the uptake of the NHS Health Check. Public Health England and Local Authority partners remain committed to offering the NHS Health Check to those aged 40-74 years old. The programme previously explored alternative points of delivery - such as community pharmacists. This piece discusses and reflects on the efforts within Manchester to use skill mix in primary dental care services and widen access to target individuals eligible for an NHS Health Check. The pilot schemes in Manchester illustrated the willingness and enthusiasm for primary care dentists to embrace change and work alongside new partners to deliver patient benefit beyond the provision of dental care. However, substantial barriers to implementation prevented the desired level of progress.

The NHS Health Check programme was introduced in England in 2009 with the intention of prioritising a risk-awareness, assessment and management programme for diabetes, chronic kidney disease, cardiovascular disease and stroke risk in adults aged 40-75. It aims to reduce morbidity and preventable deaths, with a focus on prevention and early intervention through a healthcare public health approach recommended by the Department of Health ${ }^{1}$ following the principles set out in the Ottawa Charter, ${ }^{2}$ enabling individuals and communities to obtain and sustain good health. Reducing avoidable premature mortality is seen as a Government priority and the NHS Health Check meets the objectives of $A$ call to action to reduce premature mortality ${ }^{3}$ and the Cardiovascular disease outcome strategy. ${ }^{4}$

\footnotetext{
Speciality Registrar/Honourary Lecturer in Dental Public Health, University of Manchester School of Dentistry, JR Moore Building, Manchester, M13 9PL: ${ }^{2}$ Public Health Nurse Specialist, Public Health Manchester, PO Box 532, Town Hall Extension, Manchester, M60 2LA; ${ }^{3}$ General Dental Practitioner and Chair of NHS England Greater Manchester Local Dental Network, 4th Floor, 3 Piccadilly Place, London Road Manchester, M1 3BN; ${ }^{4}$ National Lead - NHS Health Check and Blood Pressure Programme, Public Health England Health and Wellbeing Directorate, 2nd Floor, Skipton House, 80 London Road, London, SE1 6LH; ${ }^{5}$ Consultant in Dental Public Health Public Health England, 4th Floor, 3 Piccadilly Place, London Road, Manchester, M1 3BN ${ }^{*}$ Correspondence to: Dr M. G. McGrady Email: michael.mcgrady@manchester.ac.uk Tel: +44 (0)1612756689
}

\section{Refereed Paper}

Accepted 10 June 2015

DOI: $10.1038 /$ sj.bdj.2015.590

${ }^{\oplus}$ British Dental Journal 2015; 219: 107-109
Diabetes, cardiovascular and kidney disease are accountable for a significant proportion of the difference in life expectancy between affluent and deprived communities. The rise in levels of obesity and type II diabetes are becoming a major burden to healthcare resources in England and a probable risk to the system in the future. ${ }^{5}$ Therefore raising awareness, early detection and prevention are key drivers for the programme. Primary care trusts began a phased implementation in April 2009.

Following the Health and Social Care Act 2012, the responsibility for the risk assessment and life style interventions for the NHS Health Check programme sits with local authorities, funded through the public health budget. The commissioning of the risk assessment component is a mandatory function for local authorities and is a key priority in the public health outcomes framework. ${ }^{6}$

Local authorities have a legal duty to make arrangements for:

- Each eligible person aged 40-74 to be offered a NHS Health Check once every five years and for each person to be recalled every five years if they remain eligible

- The risk assessment to include specific tests and measurements

- Ensuring the person having their health check is told their cardiovascular risk score, and other results are communicated to them

- Specific information and data to be recorded and, where the risk assessment is conducted outside the person's GP practice, for that information to be forwarded to the person's GP.

The programme had been designed to enable the NHS Health Check offer to be provided under a variety of settings, such as general medical practice and pharmacies, in a community delivery model. Therefore the risk assessment could be offered in a point of care testing (POCT), or near patient testing (NPT) scenarios. During the risk assessment a number of tests and measures are performed. Where the risk assessment is conducted outside the person's GP practice, there is also a legal duty for the following information to be forwarded to the person's GP:

- Age

- Gender

- Smoking status

- Family history of coronary heart disease

- Ethnicity

- Body mass index (BMI)

- Cholesterol level

- Blood pressure

- Physical activity level - inactive, moderately inactive, moderately active or active

- Cardiovascular risk score - QRISK ${ }^{\circledR} 2^{7}$ or Framingham $^{8}$

- Alcohol use disorders identification test (AUDIT) score.

The responsibilities for follow-up and additional testing lies with primary care services funded through NHS England. Therefore there is a need for collaboration across the healthcare system between Public Health England, NHS England and 
the Health and Wellbeing Boards. The uptake of NHS Health Check offers in England has been lower than desired and has faced criticism in the media as to its value. ${ }^{8}$ Much of the evidence for the criticism was based on a Cochrane systematic review. ${ }^{9}$ Public Health England responded, stating the findings of the review were not fully applicable to the NHS Health Check programme, but agreed evidence was needed to evaluate its outcomes. Imperial College London and Queen Mary University have been awarded Department of Health funding to carry out a 'National evaluation of the NHS Health Check programme' to determine if the programme is achieving the modelled benefits described in the original policy proposals. ${ }^{10}$

In Manchester, the uptake of the NHS Health Check offer is illustrated in Figure 1. The Greater Manchester Public Health Network wanted to improve the access to and uptake of the programme.

Manchester local authority public health team commissioned the One Stop Health Bus, a dedicated mobile clinic to deliver the NHS Health Check offering targeted and opportunistic testing for all eligible individuals who want support and advice on health. The bus was commissioned to help boost numbers of checks delivered, and accessed hard to reach communities across the city. However, other methods of delivery needed to be explored to further increase uptake for target populations.

Initial discussions were held with the local authority public health team, Greater Manchester Public Health England dental public health team and a local primary care dental provider to explore the opportunities and added value a NHS Health Check offer in a dental setting could provide.

It was agreed that the target population of 'apparently well' adults were more likely to attend the dentist than their general medical practitioner in any given year. This would address one of the potential drawbacks of reliance on checks in primary medical care. The dental teams are already accustomed to delivery of public health messages through Delivering better oral health ${ }^{11}$ and the links of oral disease to general health through a common risk factor approach.

Epidemiological data demonstrates the links between the general and oral health statistics. The potential for accessing hard to reach groups through a dental practice setting would provide an opportunity to widen access to the offer of the NHS Health Check in keeping with a community delivery model in a healthcare public health context. It would also demonstrate the ability of primary care dental practice to put 'its shoulder to the wheel' in supporting health promotion on a broader scale through a common

\section{Table 1 Summary of the aims of the NHS Health Check}

'The NHS Health Check programme offers a fantastic opportunity to help people to live longer, healthier lives. It aims to improve health and wellbeing of adults aged 40-74-years through the promotion of earlier awareness, assessment and management of the major risk factors and conditions driving premature death, disability and health inequalities in England.'

\section{NHS Health Check has three components:}

Risk assessment

Risk awareness

Risk management

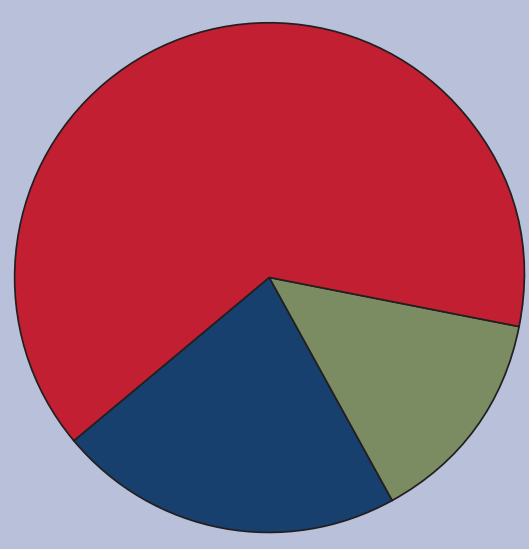

Outstanding $(67,460)$

Received $(14,531$

Offered (22,890 - ex. received)

\begin{tabular}{|c|c|}
\hline 2013-2018 (5 year cumulative) & \\
\hline Total eligible population 2013-2018 & 104,881 \\
\hline Number of people who were offered a NHS Health Check & $37,421(35.7 \%)$ \\
\hline Number of people that received a NHS Health Check & $14,531(13.9 \%)$ \\
\hline Percentage of people that received a NHS Health Check of those offered & $38.8 \%$ \\
\hline
\end{tabular}

Fig. 1 5-year cumulative data for NHS Health Checks in Manchester.

Source: www.nhshealthcheck.nhs.uk

risk factor approach and could make dental teams more aware of a population focus and whole patient care.

A service specification was drawn up setting out responsibilities and accountability, the competencies to be achieved by dental care professionals (DCPs) delivering the NHS Health Check. A training programme was devised, tailored to meet the skill set of DCPs. It was recognised they would need complementary knowledge to deliver the check offer effectively. The training days consisted of:

- A full day session on cardiovascular risk assessment and theory

- Motivational interviewing ${ }^{12}$ - theory and application

- Training on practical POCT training.

Quality assurance was performed to ensure checks were delivered within an appropriate setting and the requisite equipment and consumables available to a consistent standard. Public Health England defined quality controls were put in place and the practice was assessed by the infection control lead.

Dental teams reported that there was added value from taking part in the project as the training received, within the NHS Health Check programme, enhanced the delivery of health promotion messages from DBOH within dental care delivery. The motivational interviewing was highlighted as a significant tool that had benefited the dental team. There were perceived benefits from the community delivery model, strengthening the relationship between the dental practice the community and patients.

There were, however, significant barriers to implementation. Differences in funding arrangements between primary dental care and primary medical care lead to issues 
relating to remuneration. The early learning suggested the value placed for remuneration of checks in a dental practice was too low for it to be a viable or attractive offer in the long term. The payment to a dental practice for offering and completing checks needed to include the practice overheads, costs of consumables and administration time to submit findings, as well as a small margin of profit otherwise the offer of the NHS Health Check would not be a viable or attractive proposal for dental practices as this activity does not contribute to delivery of the dental contract. Payments for staff time to take part in training also need to be factored into the equation. Furthermore, it would need to be equitable to other healthcare providers and additional space maybe required so that taking part does not induce risk to the practice in delivery of dental services.

In addition, it became clear from dental indemnity organisations as the delivery of the NHS Health Check was not part of delivery of dental services as such dental indemnity cover was not considered to extend to any perceived risk in delivery of the NHS Health Check. This has provided an opportunity to stimulate discussion with indemnity organisations in order to ensure appropriate cover can be arranged for this programme but it also raises the question if existing indemnity arrangements should be revisited. Dental care professionals delivering extended activities are covered under a dental provider's cover but does that include activities such as fluoride varnish application outside the surgery setting?

From the feedback of the practices involved, it is clear there needs to be a better understanding by all stakeholders on the fundamental differences between the practicalities and logistics of delivery in a primary dental care practice as opposed to primary medical care setting. Better understanding of primary dental care contracting and how it differs from primary medical care. Improved communication is required between the practices and the local authority public health team.

The concept of using the NHS Health Check in a dental setting, using a community delivery model still sits within the healthcare public health paradigm, but the outcomes need to be more fully evaluated to determine its success and sustainability. Solutions for barriers to implementation need to be found in relation to indemnity and remuneration.

Offering the NHS Health Check in a dental practice setting is not suitable for all practices. The practices in the pilot were carefully selected in areas of high need and low uptake of checks, practices where the providers understood the importance of prevention and common risks and involved engagement with medical colleagues. The commissioning of dental service contract must be viewed and is entirely separate to any arrangement entered into with the local authority for health checks. Dental service activity, service delivery and performance should be unaffected. The NHS Health Check is an additional commission and service to be offered if capacity and resource are available over and above the delivery of dental services.

There was recognition of the potential benefits to patients and the increased knowledge base and use of skill mix for the dental team particularly the motivational interviewing training DCPs received to give personalised oral health advice. It was also noted that the dental care professionals were often members of the locality of the dental practice and therefore well placed to be local community assets and stimulate interest and uptake of checks within their local contact group of friends and family. Primary care practitioners have previously shown a willingness to engage and inform progress. ${ }^{13-15}$ This is an example of highly motivated primary care practitioners engaging in an agenda to increase the profile, value and skills of the dental workforce to support partners in other organisations.

Improved engagement and process modification in response to these findings are essential if we are to retain the interest and expertise of these groups of primary care clinicians and offer NHS Health Checks within dental practices. More information on the NHS Health Check programme can be found at www.healthcheck.nhs.uk.
1. Department of Health. Guidance to support the provision of healthcare public health advice to clinical commissioning groups. 2012. Online information available at https://www.gov.uk/ government/uploads/system/uploads/attachment data/file/216835/Healthcare-Public-Health-AdviceService-Guidance-FINAL.pdf (accessed July 2015).

2. World Health Organisation. Ottawa charter for health promotion. 1986. Online information available at http://www.who.int/healthpromotion/conferences/ previous/ottawa/en/ (accessed July 2015).

3. Department of Health. Living well for longer: a call to action to reduce aviodable premature mortality. 2013. Online information available at https:// www.gov.uk/government/uploads/system/uploads/ attachment_data/file/181103/Living_well_for_ longer.pdf (accessed July 2015).

4. Department of Health. Cardiovascular disease outcomes strategy. 2013. Online information available at https://www.gov.uk/government/ uploads/system/uploads/attachment_data/ file/214895/9387-2900853-CVD-Outcomes_web1. pdf (accessed July 2015).

5. Hex N, Bartlett C, Wright D, Taylor M, Varley D. Estimating the current and future costs of Type 1 and Type 2 diabetes in the UK, including direct health costs and indirect societal and productivity costs. Diabet Med 2012; 29: 855-862.

6. Department of Health. Improving outcomes and supporting transparency. Part 1: a public health outcomes framework for England, 2013-2016. 2012. Online information available at https://www.gov.uk/ government/uploads/system/uploads/attachment_ data/file/263658/2901502_PHOF_Improving_

Outcomes_PT1A_v1_1.pdf (accessed July 2015).

7. Collins $G \bar{S}$, Altman $\bar{D}$ G. Predicting the 10 year risk of cardiovascular disease in the United Kingdom: independent and external validation of an updated version of QRISK2. BMJ 2012; 344: e4181.

8. Gould M. Expert panel will assess cost effectiveness of health checks. BMJ 2013; 347: f5222.

9. Krogsboll L T, Jorgensen K J, Gronhoj Larsen C, Gotzsche P C. General health checks in adults for reducing morbidity and mortality from disease. Cochrane Database Syst Rev 2012; 10: CD009009.

10. Department of Health. Economic modelling for vascular checks. 2008. Online information available at http://www.em-online.com/download/medical article/36931_DH_085917.pdf (accessed July 2015).

11. Public Health England. Delivering better oral health: an evidence-based toolkit for prevention. Third edition. 2014. Online information available at https://www.gov.uk/government/uploads/ system/uploads/attachment_data/file/367563/ DBOHv320140CTMainDocument_3.pdf (accessed July 2015).

12. Rollnick S, Miller W R, Butler C. Motivational interviewing in health care: helping patients change behavior. New York: Guilford Press, 2007.

13. Brocklehurst $P$, Bridgman $C$, Davies G. A qualitative evaluation of a Local Professional Network programme 'Baby Teeth DO Matter'. Community Dent Health 2013: 30: 241-28.

14. Harris R, Bridgman C. Introducing care pathway commissioning to primary dental care: the concept. BrDent J 2010; 209: 233-239.

15. Harris $R$, Bridgman $C$, Ahmad M et al. Introducing care pathway commissioning to primary dental care measuring performance. Br Dent J 2011; 211: E22. 